\title{
Computational Insights into the Competitive Inhibition of Acetyl Coenzyme A and Succinyl Coenzyme A of the First Step of Citric Acid Cycle
}

Salam Pradeep Singh* and Bolin Kumar Konwar

Department of Molecular Biology and Biotechnology, Tezpur University, India.

\begin{abstract}
Citric acid cycle comprises a various chemical reactions and it is required by all aerobic organisms to generate ATP. The present investigation focuses on the competitive inhibition of citrate synthase- the first step of the citric acid cycle. The known natural substrate of citrate synthase is acetyl Coenzyme A. Initially, the first substrate oxaloacetate binds to the citrate synthase which then induces the enzyme to change its conformation thus creating a binding site for the acetyl Coenzyme A.
\end{abstract}

There are also several reports of citrate synthase enzyme inhibited by succinyl Coenzyme A which resembles acetyl Coenzyme $A$ and acts as a competitive inhibitor. Hence, the present investigation deals with the molecular docking simulation studies of the two substrates viz. acetyl Coenzyme A and succinyl Coenzyme $A$ at the active site of the citrate synthase to understand the insights into the competitive inhibition of these two substrates. Lastly, we have also performed the density functional theory (DFT) analysis of acetyl Coenzyme A and succinyl Coenzyme A to understand the atomic charge that might contribute in the competitive inhibition.

The molecular docking scores and interaction energy revealed acetyl Coenzyme A showing competitive inhibition with succinyl Coenzyme A with favourable energy. Also the DFT studies revealed the plausible caused of the competitive inhibition at the atomic level.

Keywords: Molecular docking; DFT; Acetyl Coenzyme A and succinyl Coenzyme A

\section{Introduction}

The citric acid or the Krebs cycle, [1] comprises a series of chemical reactions utilized by all aerobic organisms to generate its energy through the oxidation of acetate derived from carbohydrates, fats and proteins [2]. The final outcome of the cycle releases carbon dioxide and chemical energy in the form of adenosine triphosphate (ATP) [3]. The citric acid is one of the earliest established components of cellular metabolism and it may have originated abiogenically [4]. The citric acid cycle begins with the transfer of a two-carbon acetyl group from acetyl Coenzyme A to the four-carbon acceptor compound (oxaloacetate) to form a six-carbon compound (citrate) [5]. The enzyme citrate synthase catalyzes the condensation reaction of the two-carbon acetate residue from acetyl Coenzyme A and a molecule of four-carbon oxaloacetate to form the six-carbon citrate [5]. The enzyme citrate synthase is present in all living cells and stands as a pace-making enzyme in the first step of the Citric Acid Cycle [1]. Citrate synthase is localized within eukaryotic cells in the mitochondrial matrix, and it is commonly used as a quantitative enzyme marker for the presence of intact mitochondria.

Oxaloacetate - the first substrate which binds to the citrate synthase induces the enzyme to change its conformation thereby creating a binding site for the acetyl Coenzyme A. Citrate synthase consists of 437 amino acid residues are organized into two main subunits, each consisting of 20 alpha-helices. These alpha helices compose approximately $75 \%$ of citrate synthase's tertiary structure. Between these two subunits, a single cleft exists containing the active site. Two binding sites can be found therein: one reserved for citrate or oxaloacetate and the other for Coenzyme A. The active site consists of three key residues i.e. His274, His320, and Asp375 that are highly selective in their interactions with substrates [6]. The enzyme is also inhibited by succinyl Coenzyme A, which resembles acetyl Coenzyme $\mathrm{A}$ and acts as a competitive inhibitor $[7,8]$. There are also reports of the inhibition of citrate synthase by acetyl Coenzyme A analogues which prove the existence of a single active site [9-11]. The present investigation focus on the computational insights into the competitive inhibition of acetyl Coenzyme A and succinyl Coenzyme $A$ at the active site of citrate synthase using molecular docking simulation approaches and density functional theory (DFT) analysis of acetyl Coenzyme A and succinyl Coenzyme A.

\section{Materials and Method}

\section{Protein preparation}

The three dimensional crystal structure of citrate synthase complexed with oxaloacetate (PDB ID: 4CTS) was retrieved from the Protein Data Bank (http://www.rcsb.org/). The crystal structure has resolution of $2.90 \AA$ and a structural weight of $98217.33 \mathrm{Da}$. It has an amino acid length of 437 and contains two chains (Chain A and B). The enzyme was then imported in the Molegro Virtual Docker (MVD) [12]. For molecular docking purpose, all the water molecules were removed because they are considered during the scoring while the complex ligand oxaloacetate was also imported in MVD. For docking purposes, the active site residues (His274, His 320 and Asp 375) were set as the search space. The active site residues of search space were set

*Corresponding author: Salam Pradeep Singh, Department of Molecular Biology and Biotechnology, Tezpur University, Tezpur-784028, Assam, India, Tel: 91 3712267007/5432; E-mail: salampradeep@gmail.com

Received October 27, 2012; Accepted December 16, 2012; Published December 18,2012

Citation: Singh SP, Konwar BK (2012) Computational Insights into the Competitive Inhibition of Acetyl Coenzyme A and Succinyl Coenzyme A of the First Step of Citric Acid Cycle. Bioenergetics 2: 109. doi:10.4172/2167-7662.1000109

Copyright: $\odot 2012$ Singh SP, et al. This is an open-access article distributed unde the terms of the Creative Commons Attribution License, which permits unrestricted use, distribution, and reproduction in any medium, provided the original author and source are credited. 
Citation: Singh SP, Konwar BK (2012) Computational Insights into the Competitive Inhibition of Acetyl Coenzyme A and Succinyl Coenzyme A of the First Step of Citric Acid Cycle. Bioenergetics 2: 109. doi:10.4172/2167-7662.1000109

Page 2 of 4

inside a restriction sphere of radius $15 \AA$ (X: 36.81, Y: -2.68, Z: 10.12) using MVD. The side chain flexibility of the active site residue of the enzyme (His274, His 320 and Asp 375), set with a tolerance of 1.10 and strength of 0.80 for docking simulations.

\section{Chemical dataset}

The 2D structures of acetyl Coenzyme A and succinyl Coenzyme A was retrieved from the NCBI PubChem database [13]. The energy of these compound were optimized using MM2 force field methods implemented in ChemOffice 2010 (ChemOffice 2010: CambridgeSoft Corporation) and converted to its corresponding three dimensional format and save as sybyl mol2 file format for docking purposes.

\section{DFT Optimization}

The 3D geometrical structures of acetyl Coenzyme A and succinyl Coenzyme A was further optimized using DFT using General Atomic and Molecular Electronic Structure System (GAMESS) [14] implemented in ChemOffice 2010. The DFT analysis uses since quantum chemical calculationsin determining the molecular structure which will aid in understanding the intermolecular interactions of the competitive inhibition of the enzyme. The DFT B3LYP/6-31G basis set was used for the DFT calculation [15].

\section{Molecular docking}

In the present investigation, molecular docking simulation was carried out using Molegro Virtual Docker. The software is based on a differential evolution algorithm; the solution of the algorithm considers the sum of the intermolecular interaction energy between the ligand and the protein and the intramolecular interaction energy of the ligand. The docking energy scoring function is based on the modified piecewise linear potential (PLP) with new hydrogen bonding and electrostatic terms included [16].

The docking algorithm was set with softens potentials during the docking simulation with the side chains of the enzyme made being flexibility. This is because flexible docking is considered to be more reliable and accurate than rigid docking. The maximum minimization for the residues and the ligand was set at 2000 steps and the maximum global minimization was set for 2000 steps.

The MolDock scoring function was also set with a grid resolution of $0.30 \AA$ and a maximum iteration of 1,500 with a simplex evolution size of 50 and a minimum of 20 runs were performed for the two compounds with threshold energy of 100 . Also, the simplex evolution was set for 300 steps with a neighbour distance factor of 1.00. Finally, the best pose of the two compounds was selected for the subsequent ligand-protein interaction energy analysis.

\section{Results}

Molecular docking simulation was carried out using MVD. The docking scores for acetyl Coenzyme A and Succinyl Coenzyme A including the interaction energy and hydrogen bonding energy are shown in Table 1. Additionally, the molecular interaction analysis for the ligand-protein interaction including the interaction distance and interaction energy of acetyl Coenzyme A and succinyl Coenzyme A is shown in Table 2. Also, the snapshots of ligand-protein interaction depicting the binding mode of acetyl Coenzyme A and succinyl Coenzyme A are shown in Figure 1A and Figure 2A. The non bonded electrostatic interaction of acetyl Coenzyme A and succinyl Coenzyme A are shown in Figure 1B and Figure 2B respectively.

\begin{tabular}{|l|l|l|l|l|l|l|}
\hline SN & Compound & $\begin{array}{l}\text { MolDock } \\
\text { Score }\end{array}$ & Rerank Score & Interaction & Internal & HBond \\
\hline 1 & $\begin{array}{l}\text { Acetyl } \\
\text { Coenzyme A }\end{array}$ & -127.339 & -109.21 & -124.03 & -3.308 & -14.10 \\
\hline 2 & $\begin{array}{l}\text { Succinyl Co- } \\
\text { enzyme A }\end{array}$ & -119.684 & -98.11 & -111.73 & -7.954 & -12.98 \\
\hline
\end{tabular}

Table 1. Docking scores of acetyl Coenzyme A and succinyl Coenzyme A
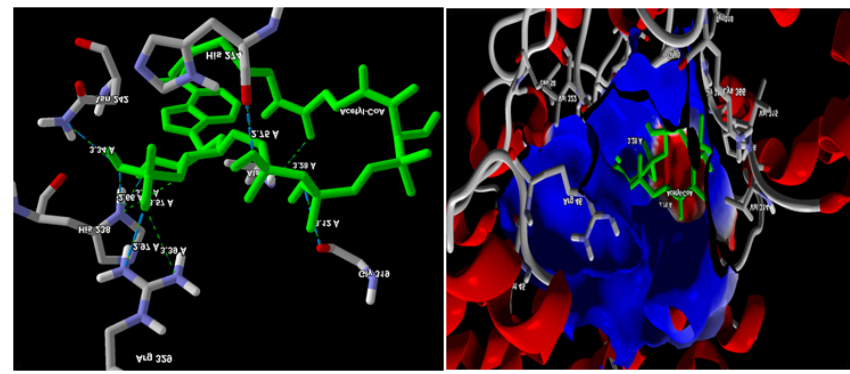

Figure 1: (A) Molecular interaction depicting acetyl Coenzyme $A$ at the active site of citrate synthase and (B) Non bonded electrostatic interaction depicting acetyl Coenzyme $A$ at the active site of citrate synthase.
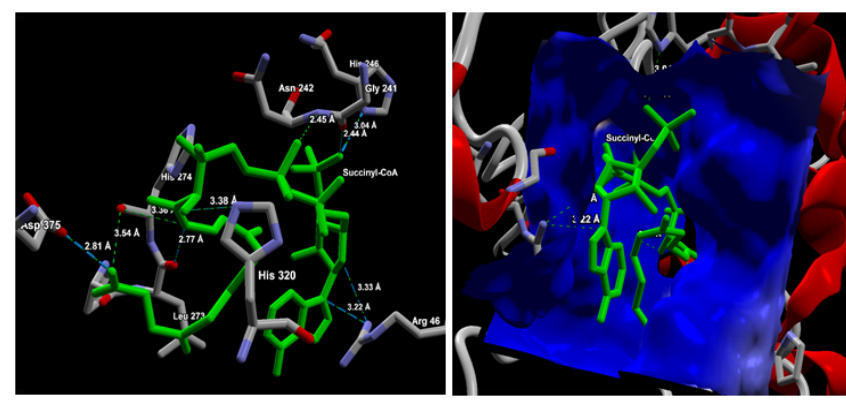

Figure 2: (A) Molecular interaction depicting succinyl Coenzyme $A$ at the active site of citrate synthase and (B) Non bonded electrostatic interaction depicting succinyl Coenzyme $A$ at the active site of citrate synthase.

Lastly, the Mülliken atomic charges (MAC) calculated using GAMESS for acetyl Coenzyme A and succinyl Coenzyme A at DFT B3LYP/6-31G basis set is shown in Figure 3.

\section{Discussion}

The docking scores revealed acetyl Coenzyme A showing competitive inhibition with succinyl Coenzyme A (Table 1) with favourable rerank score and MolDock score. The rerank score used in MVD is a weighted combination of the terms used by the MolDock score mixed with a few addition terms which includes the Steric terms which are Lennard-Jones approximations to the steric energy [16]. It is computationally more expensive than the scoring function but it is generally gives better result than the docking score function. The reranking coefficients used the energy parameters such as E-Inter total, E-Inter (protein-ligand), Steric, VdW (Van der Waal's), HBond (hydrogen bonding energy), E-Intra (tors, ligand atoms), E-Solvation, E-Total etc.

Both acetyl Coenzyme A and succinyl Coenzyme A were found to be lying deep inside the active site of citrate synthase exhibiting both bonded and non bonded interaction.

The interaction energy of acetyl Coenzyme A is - $124.03 \mathrm{~kJ}$ mol-1 compared to $-111.73 \mathrm{~kJ}$ mol-1 of succinyl Coenzyme A. While hydrogen 
Citation: Singh SP, Konwar BK (2012) Computational Insights into the Competitive Inhibition of Acetyl Coenzyme A and Succinyl Coenzyme A of the First Step of Citric Acid Cycle. Bioenergetics 2: 109. doi:10.4172/2167-7662.1000109

Page 3 of 4

\begin{tabular}{|c|c|c|c|c|}
\hline $\mathrm{SN}$ & Ligand & Protein-Ligand Interaction & Interaction Distance & Interaction Energy (kJ mol-1) \\
\hline \multirow[t]{9}{*}{1} & \multirow{9}{*}{ Acetyl Coenzyme A } & His274(O)....... $(14)$ & $2.75 \AA$ & -2.5 \\
\hline & & Gly319(O)......O(16) & $3.12 \AA$ & -2.3 \\
\hline & & $\operatorname{Arg} 329(\mathrm{NH} 2) \ldots \ldots . \mathrm{O}(8)$ & $3.39 \AA$ & -0.4 \\
\hline & & $\operatorname{Arg} 329(\mathrm{NH} 1) \ldots \ldots . \mathrm{O}(8)$ & $2.97 \AA$ & -2.5 \\
\hline & & His328(ND1).......(9) & $2.66 \AA$ & -1.8 \\
\hline & & His328(ND1).......(5) & $3.57 \AA$ & -0.05 \\
\hline & & His328(ND1).......O(6) & $3.11 \AA$ & -1.2 \\
\hline & & Asn242(ND2).......(9) & $3.34 \AA$ & -1.3 \\
\hline & & Ala321(N),.....O(19) & $3.28 \AA$ & -0.46 \\
\hline \multirow[t]{10}{*}{2} & \multirow[t]{10}{*}{ Succinyl } & Asp375(OD1).......(21) & $2.81 \AA$ & -2.5 \\
\hline & & His $274(\mathrm{O}) \ldots \ldots . \mathrm{O}(21)$ & $3.54 \AA$ & -0.3 \\
\hline & & His $274(0) \ldots \ldots . . N(28)$ & $3.36 \AA$ & -0.3 \\
\hline & & Leu273(O).......N(28) & $2.77 \AA$ & -1.5 \\
\hline & & His320(NE2)....... O(14) & $3.38 \AA$ & -1.0 \\
\hline & & $\operatorname{Arg} 46(\mathrm{NH} 1) \ldots \ldots . \mathrm{O}(4)$ & $3.33 \AA$ & -1.3 \\
\hline & & $\operatorname{Arg} 46(\mathrm{NH} 1) \ldots \ldots . . \mathrm{N}(23)$ & $3.22 \AA$ & -1.9 \\
\hline & & Asn242(N)...... O(16) & $2.45 \AA$ & -0.03 \\
\hline & & Gly241(O).......(9) & $2.44 \AA$ & -1.2 \\
\hline & & His246(ND1)...... O(9) & $3.04 \AA$ & -2.5 \\
\hline
\end{tabular}

Table 2. Molecular interaction analysis of acetyl Coenzyme $A$ and succinyl Coenzyme $A$ at the active site citrate synthase

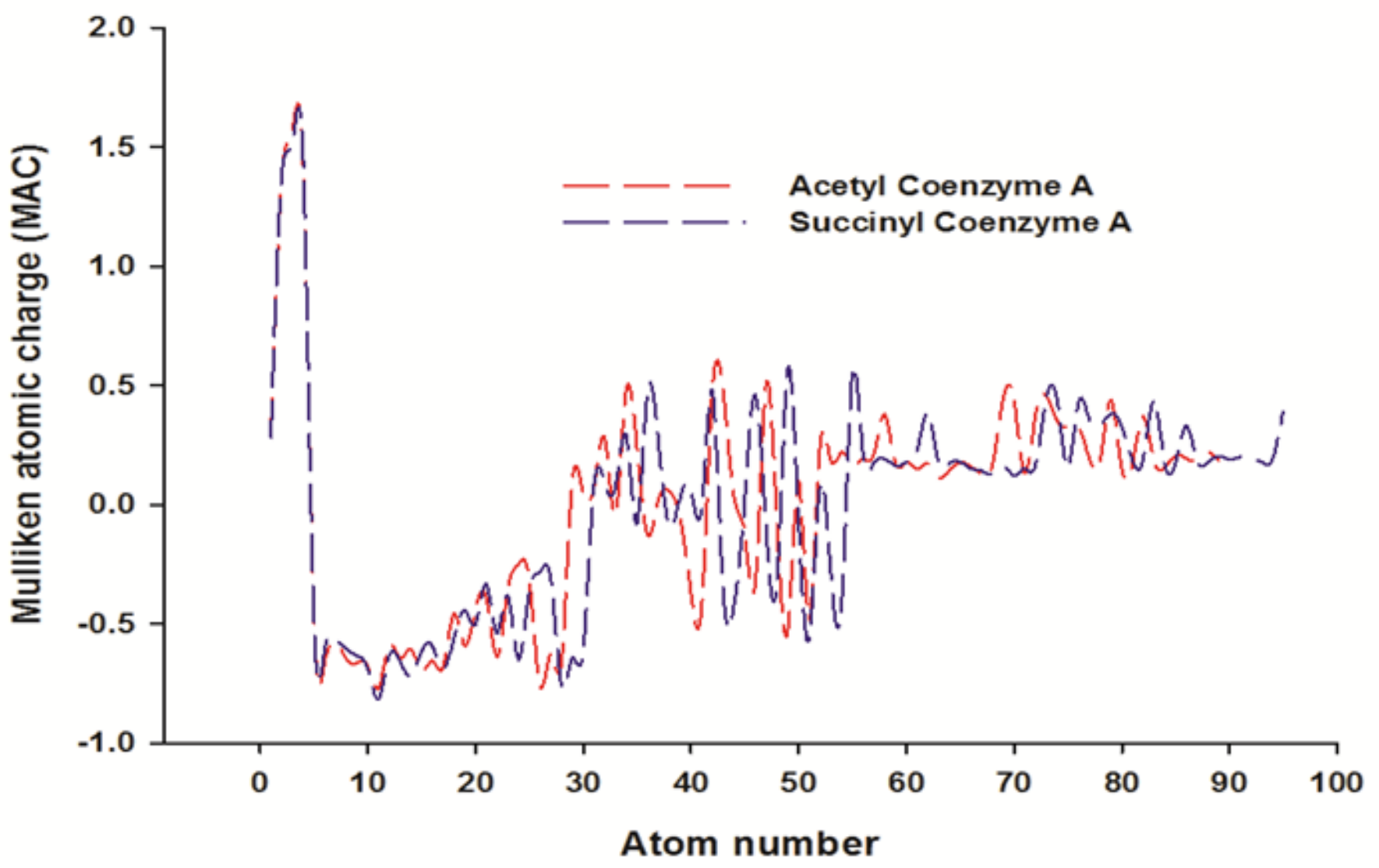

Figure 3. Mulliken atomic charge (MAC) plot of acetyl Coenzyme A and succinyl Coenzyme A calculated at DFTB3LYP/6-31G basis set. 
Citation: Singh SP, Konwar BK (2012) Computational Insights into the Competitive Inhibition of Acetyl Coenzyme A and Succinyl Coenzyme A of the First Step of Citric Acid Cycle. Bioenergetics 2: 109. doi:10.4172/2167-7662.1000109

Page 4 of 4

bonding interaction energy which accounts for the free binding energy of acetyl Coenzyme A is $-14.10 \mathrm{~kJ}$ mol- 1 compared to- $12.98 \mathrm{~kJ}$ mol-1 of succinyl Coenzyme A.

From Table 2, it is revealed that Acetyl Coenzyme A showed molecular interaction with His274(O), Gly319(O), Arg329(NH2), Arg329(NH1), His328(ND1), Asn242(ND2) and Ala321(N) residues of citrate synthase. While succinyl Coenzyme A establish molecular interaction with Asp375(OD1), His 274(O), Leu273(O), His320(NE2), $\operatorname{Arg} 46(\mathrm{NH} 1)$, Gly241(O), Asn242(N) and His246(ND1) residues of citrate synthase. The top three docking hits showed common molecular interaction with Asp93 (OD2).

From Figure 3 The atomic charge densities are indicates the atomic positions which might contribute in the competitive inhibition of acetyl Coenzyme A and succinyl Coenzyme at the active site of citrate synthase.

\section{Conclusion}

We have performed the molecular interaction analysis of acetyl Coenzyme A and succinyl Coenzyme at the active site of citrate synthase - the first step reaction of the citric acid cycle in order to understand the competitive inhibition of acetyl Coenzyme A and succinyl Coenzyme. The molecular docking simulation and the molecular interaction analysis revealed that the favourable interaction energy and the hydrogen bonding energy of acetyl Coenzyme A accounts competitive inhibition with succinyl Coenzyme A. We have also depicted the binding mode of acetyl Coenzyme A and succinyl Coenzyme A in Figure 1 and Figure 2. In fact, acetyl Coenzyme A mostly interact with the donor atom of the interacting amino acids of citrate synthase which is the major driving force in showing acetyl Coenzyme A showing competitive with succinyl Coenzyme A at the active site of citrate synthase.

\section{Acknowledgement}

The author's thank Apex Bioinformatics, Dept. of Biotechnology, Ministry of Science and Technology, New Delhi, India for promoting Bioinformatics in North Eastern part of India.

\section{References}

1. Krebs HA, Weitzman PDJ (1987) Krebs' citric acid cycle: half a century and still turning. London: Biochemical Society.

2. Lowenstein JM (1969) Methods in enzymology: citric acid cycle (Vol13) Academic Press, Boston, USA.

3. Lane, Nick (2009). Life Ascending: The Ten Great Inventions of Evolution. New York: W.W. Norton \& Co.

4. Gest $\mathrm{H}$ (1987) Evolutionary roots of the citric acid cycle in prokaryotes. Biochem Soc Symp 54: 3-16.

5. Wiegand G, Remington SJ (1986) Citrate Synthase: Structure, Control, and Mechanism. Annu Rev Biophys Biophys Chem 15: 987-117.

6. Lehninger (2005) Principles of biochemistry. (4th Edn), W.H. Freeman and Co.

7. Bayer E, Bauer B, Eggerer H (1981) Evidence from Inhibitor Studies for Conformational Changes of Citrate Synthase. Eur J Biochem 120: 155-160.

8. Selwood T, Jaffe EK (2011) Dynamic dissociating homo-oligomers and the control of protein function. Arch Biochem Biophys 519: 131-43

9. Meléndez-Hevia E, Waddell TG, Cascante M (1996). The puzzle of the Krebs citric acid cycle: assembling the pieces of chemically feasible reactions, and opportunism in the design of metabolic pathways during evolution. $\mathrm{J}$ Mol Evo 43: 293-303.

10. Voet D, Voet JG (2004). Biochemistry (3rd ed.). New York: John Wiley \& Sons, Inc. p. 615.

11. Johnson JD, Mehus JG, Tews K, Milavetz BI, Lambeth DO (1998). Genetic evidence for the expression of ATP- and GTP-specific succinyl-CoA synthetases in multicellular eucaryotes. J Biol Chem 273: 27580-27586.

12. Molegro APS (2011) MVD 5.0 Molegro Virtual Docker. Aarhus C, Denmark.

13. Bolton E, Wang Y, Thiessen PA, Bryant SH (2008) PubChem: integrated platform of small molecules and biological activities. In: Annual reports in computational chemistry $4: 217-241$.

14. Schmidt MW, Baldridge KK, Boatz JA, Elbert ST, Gordon MS et al. (1993) General atomic and molecular electronic structure system. J Comp Chem 14 1347-1363.

15. Kim K and. Jordan KD (1994) Comparison of density functional and MP2 calculations on the water monomer and dimer. J Phys Chem 98: 10089-10094.

16. Thomsen R, Christensen MH (2006) MolDock: A New Technique for HighAccuracy Molecular Docking. J Med Chem 49: 3315-3321. 